\title{
Simulación de oleaje en los alrededores de la Isla del Coco, Costa Rica
}

\author{
Rodney E. Mora-Escalante ${ }^{1,2,3 *} \&$ Juan Pablo Ureña-Mora ${ }^{3,4}$ \\ 1. Centro de Investigación en Ciencias del Mar y Limnología (CIMAR), Ciudad de la Investigación, Universidad de \\ Costa Rica, San José, Costa Rica; rodney.moraescalante@ucr.ac.cr \\ 2. Escuela de Física, Sede Rodrigo Facio, Universidad de Costa Rica, San José, Costa Rica. \\ 3. Módulo de Información Oceanográfica (MIO), Centro de Investigación en Ciencias del Mar y Limnología, Ciudad de \\ la Investigación, Universidad de Costa Rica, San José, Costa Rica. \\ 4. Maestría en Ciencias de la Atmósfera, Programa de Posgrado en Ciencias de la Atmósfera, Universidad de Costa Rica, \\ San José, Costa Rica; juan.urenamora@ucr.ac.cr \\ * Correspondence
}

Recibido 30-I-2019. Corregido 22-V-2019. Aceptado 28-VI-2019.

\begin{abstract}
Numerical simulation of wave field around Cocos Island, Costa Rica. Introduction: periodically energetic waves, originated in the Southern Ocean, arrive to Cocos Island, because of its location in the Pacific Ocean and in deep waters. The island acts as a shelter dissipating part of the energy of the waves that reach the Pacific coast of Costa Rica. Objetive: The objective of the work is to characterize the general conditions of the swell through the numerical simulation of the swell in the Eastern Tropical Pacific (ETP), with special interest in Cocos Island. Methods: The waves are propagated with the WAVEWATCH III wave spectral model. An unstructured mesh is used in the model. Two years (2007-2008) of hindcast data are used as boundary conditions obtained from reanalysis with the WAVEWATCH model, generated by the French Institute for Marine Research (IFREMER for its acronym in French). Annual and seasonal maps and time series of significant wave height, peak period and peak wave direction are obtained. Results: The results shown are similar to other previous simulation and observation studies. The mean wave field is characterized by long periods from southwest direction. The wave conditions on the island obey the extratropical systems of both hemispheres and local processes in the PTE. Conclusions: The WAVEWATCH III wave model showed that it represents the typical wave conditions in the surroundings of Cocos Island. It is the first wave simulation work applying an unconventional mesh in the exclusive economic zone of Costa Rica. The study serves as a basis for extending to other specific areas of the coast.
\end{abstract}

Key words: significant wave height; unstructured mesh generation; Cocos Island; swell; Eastern Tropical Pacific; WAVEWATCH III.

Mora-Escalante, R. E., \& Ureña-Mora, J. P. (2020). Simulación de oleaje en los alrededores de la Isla del Coco, Costa Rica. Revista de Biología Tropical, 68(Supl. 1), S198-S212.

La Isla del Coco emerge en el Pacífico Tropical del Este (PTE) como una provincia marina, es decir, un monte submarino ubicada sobre la Cordillera Volcánica del Coco (Lizano, 2008; 2012; Rojas \& Alvarado, 2012). Se localiza en las coordenadas $5.5220^{\circ} \mathrm{N}-87.0758^{\circ} \mathrm{W}$ según lo reportado por Lizano (2012) y pertenece a la provincia de Puntarenas, Costa Rica.
Desde el siglo pasado se han observado las olas superficiales que se generan por el viento y se propagan en el Océano Pacífico y que arriban a las costas de América. Los primeros trabajos de observación de oleaje se remiten a Munk \& Snodgrass (1957) en la Isla Guadalupe, México y la parte sur de California, y el de Snodgrass, Hasselmann, Miller, Munk \& 
Powers (1966) con seis estaciones de medición entre Nueva Zelanda y Alaska. Ellos concluyen de la información obtenida en las diferentes estaciones de medición, que el oleaje es de origen lejano, de baja frecuencia y se genera en el Océano del Sur. La Isla del Coco se encuentra en una de las zonas o piscinas de mayor presencia de marejadas de fondo u oleaje largo (Chen, Chapron, Ezraty, \& Vandemark, 2002; Alves, 2006) que se propagan en el PTE.

Estudios más recientes en el PTE sobre el clima marino de la región son llevados a cabo por Amador, Alfaro, Lizano \& Magaña (2006) $\&$ Lizano (2007), que mencionan que la mayor parte del oleaje climatológico en el Pacífico de Costa Rica llega del suroeste y es de origen distante. Específicamente sobre la Isla del Coco el oleaje que arriba es de dirección suroeste en promedio, de acuerdo con el análisis de Lizano (2008) con el conjunto de datos de reanálisis de WAVEWATCH III para el periodo 19972004. Actualmente no hay ningún estudio de simulación numérica de oleaje que se enfoque en el PTE, en especial sobre la Isla del Coco, Costa Rica. Algunos trabajos se enfocan en simulación de oleaje en condiciones de huracán en la región del Caribe (Lizano \& Moya, 1990; Lizano, Ocampo, Alvarado, Vega, \& Puig, 2001) y otros son de clima marino que abarcan el Océano Pacífico y otras áreas del océano (Young, 1999; Alves, 2006).

Los modelos oceánicos de circulación o los modelos de oleaje en su mayoría están definidos con base a una malla rectangular o cartesiana. Recientemente los modelos numéricos han evolucionado hacia la incorporación en su estructura interna de mallas no estructuradas para definir el dominio de configuración, por ejemplo, el modelo de circulación ADCIR (Luettich \& Westerink, 2004), el modelo de olas Simulating Waves Nearshore (SWAN, por sus siglas en inglés) (Booij, Holthuijsen, \& Ris, 1997) o la herramienta para modelado costero MIKE21 (Warren \& Bach, 1992). Existen una serie de programas de generación de mallas no estructuradas que van desde comerciales (SMS desarrollado por AQUAVEO, https://www. aquaveo.com) hasta de dominio público como
Triangle (Shewchuk, 1996), Gmsh (Geuzaine \& Remacle, 2009), Qmesh (Avdis, Candy, Hill, Kramer, \& Piggott, 2018), entre otros. Algunos estudios se basan en aplicaciones en energía renovable (Avdis et al., 2018), en aplicaciones costeras (Remacle \& Lambrechts, 2016), en la simulación de huracanes (Dietrich et al., 2012) o en la simulación de oleaje en la costa (Zijlema, 2010; Ardhuin \& Roland, 2012). La principal ventaja de usar una malla no estructurada para aplicaciones marinas es el detalle que se obtiene de la línea de costa. Por ejemplo, permite definir regiones en el dominio computacional que por los cambios abruptos en la topografía del fondo marino requieren una mayor resolución o zonas complejas en la línea de costa.

Este estudio es uno de los primeros trabajos que simulan las condiciones de oleaje en las aguas adyacentes a la Isla del Coco. Pretende mostrar características generales del campo de oleaje a partir de la simulación numérica del oleaje usando una malla no estructurada. La simulación de oleaje se lleva a cabo con la información suministrada en la frontera del modelo utilizando un conjunto de datos históricos de oleaje. Se describen tres parámetros usuales para caracterizar el oleaje: la altura significante del oleaje, el periodo y dirección del oleaje. Se muestran el comportamiento anual y estacional de estas variables típicas sobre los alrededores de la isla. También se incluye una comparación de los resultados de este estudio con el modelo operacional de oleaje WAVEWATCH III de la Administración Nacional del Océano y la Atmósfera (NOAA, por sus siglas en inglés) en un punto cercano a la isla. Los detalles del área de estudio, el programa de generación de mallas no estructuradas, el modelo de oleaje y la información de reanálisis se describen en la siguiente sección.

\section{MATERIALES Y MÉTODOS}

Área de estudio: La región de estudio comprende los alrededores de la Isla del Coco, Costa Rica. La isla emerge como un punto que sobresale en el PTE y con aguas profundas en 
sus alrededores. En la Fig. 1 se muestra el área de interés para el presente estudio. El punto más cercano a la región continental de Costa Rica, es Cabo Blanco, Puntarenas, que se ubica a una distancia aproximadamente de $500 \mathrm{~km}$ (Wyrtki, 1967; Lizano, 2001; Kessler, 2006).

\section{Generador de mallas no estructuradas:}

Se emplea el programa de elemento finito tridimensional Gmsh (Geuzaine \& Remacle, 2009) para la generación de la malla no estructurada. Es un programa de código abierto, robusto, tiene una interfaz gráfica que permite al usuario la creación y personalización de la malla en $2 \mathrm{D}$ y $3 \mathrm{D}$. El costo computacional en la generación de la malla es de alrededor de 5 segundos en un sistema operativo Linux, con procesador i7 de Intel y memoria ram de 24 GB.

Para el presente estudio se utiliza el algoritmo MeshAdapt para generar la malla final (véase la Fig. 2). La malla consiste en 2105 nodos y 4192 elementos. Para definir el tamaño de los elementos triangulares se escoge una resolución en la frontera e islas de 5.0 $\mathrm{x} 10^{3}$ y $2.0 \times 10^{-2} \mathrm{~m}$, respectivamente. Para delimitar los nodos sobre la línea de costa se utilizó la información suministrada por el Sistema de Información Geográfica Marino, Costero y Limnológico (SIGMAR) del Centro de Investigación en Ciencias del Mar y Limnología (CIMAR) de la Universidad de Costa Rica (UCR).

Datos históricos de oleaje: Se utiliza información del reanálisis de oleaje generado por el Instituto Francés para la Investigación del Mar (IFREMER por sus siglas en francés) (Rascle \& Ardhuin, 2013) con el modelo numérico WAVEWATCH III. Para el estudio se emplearon datos espectrales de oleaje en doce puntos específicos alrededor de la Isla del Coco (corresponde a los valores en la frontera de la Fig. 2, lado derecho) como condiciones de contorno. El conjunto de datos comprende solamente los años 2007 y 2008, de información espectral cada 3 horas que fueron extraídos del sitio ftp://ftp.ifremer.fr/ifremer/ ww3/HINDCAST/. También se utilizan datos históricos de oleaje del modelo operacional NOAA/WAVEWATCH III de altura del oleaje, periodo y dirección correspondiente al pico del espectro del oleaje. Este conjunto de datos fue

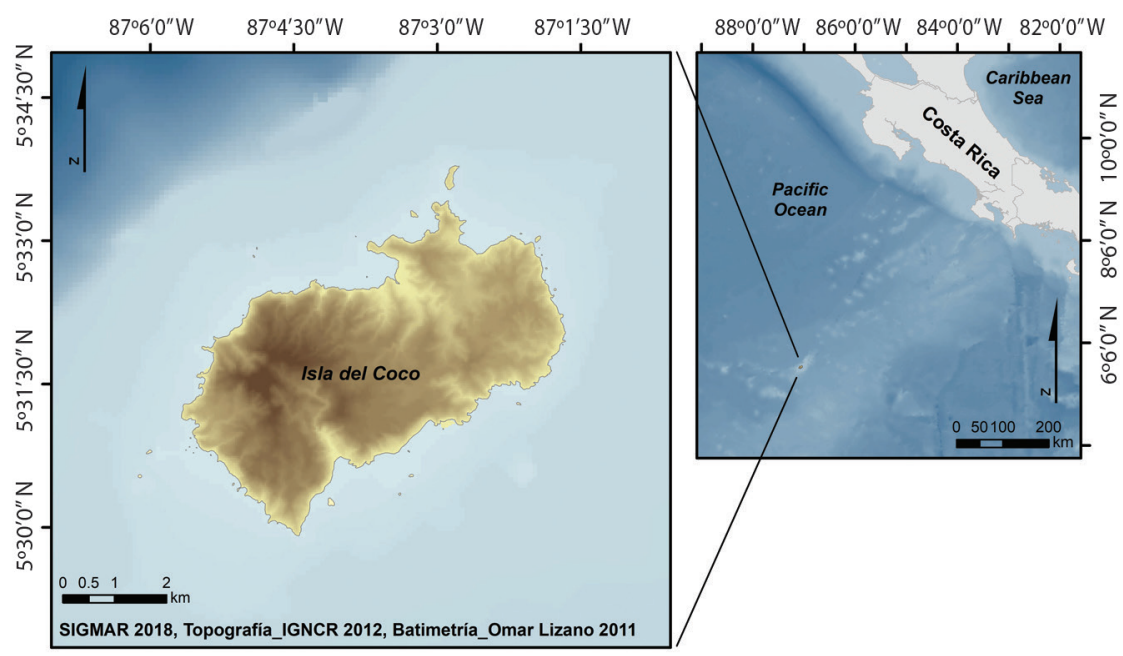

Fig. 1. Región de estudio. La figura de la izquierda muestra los alrededores de la Isla del Coco. La figura de la derecha muestra el PTE donde sobresale la Isla del Coco. Los tonos azules representan la batimetría, con los tonos claros aguas someras y los tonos oscuros aguas profundas. Fuente: SIGMAR-CIMAR, UCR.

Fig. 1. Study area. The figure on the left show the surroundings of the Cocos Island. The figure on the right shows the PTE where Cocos Island stands out. The blues shaded represent the bathymetry, with light blue are shallow waters and dark blue are deep waters. Source: SIGMAR-CIMAR, UCR. 


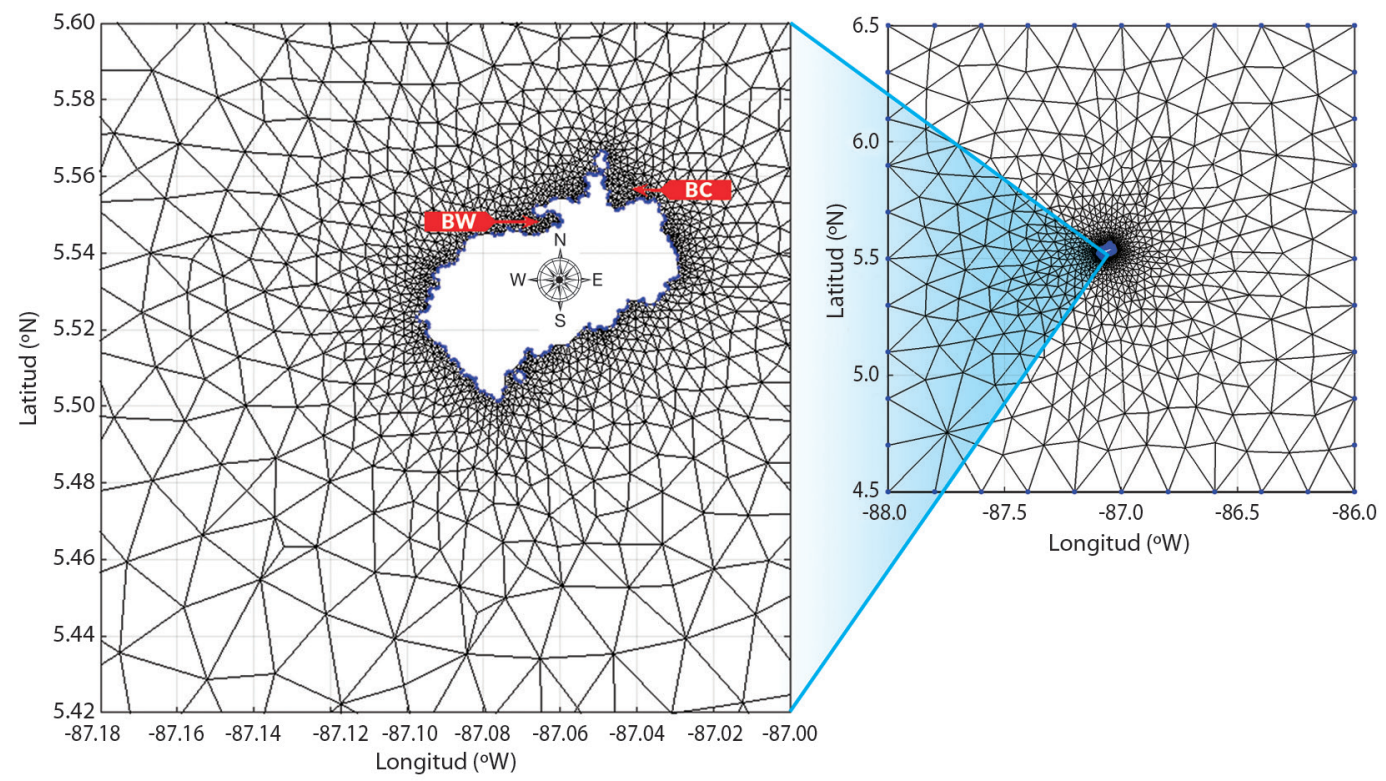

Fig. 2. Malla no estructurada para la Isla del Coco. La figura de la derecha muestra el dominio computacional utilizado en el estudio. La figura de la izquierda muestra un acercamiento sobre la isla para hacer énfasis en la mayor densidad de triángulos en la costa. Los puntos azules representan los nodos en la frontera (figura de la derecha) y la línea de costa (figura de la izquierda). Las flechas rojas muestran los sitios de Bahía Wafer (BW) y Bahía Chatham (BC).

Fig. 2. Unstructured grid for the Cocos Island. The figure on the right show computational domain uses in this study. The figure on the left show a zoom on the island to enhance the greater density of triangles on the coast. The blue points represent the nodes in the boundary (figure on the right) and the coastline (figure on the left). The red arrows show the sites of Wafer bay $(\mathrm{BW})$ and Chatham bay $(\mathrm{BC})$.

extraído del servidor ftp://polar.ncep.noaa.gov/ pub/history/waves, del Centro Nacional para la Predicción del Medioambiente (NCEP, por sus siglas en inglés).

Modelo numérico de oleaje: Se emplea el modelo espectral de oleaje WAVEWATCH III versión 4.18 (Tolman, 2014). De aquí en adelante MIOCIMAR/WAVEWATCH III. Es un modelo numérico de propagación de olas en aguas profundas. Su rango de aplicabilidad va desde el pronóstico de oleaje en aguas abiertas o en zonas costeras, estudios de oleaje generado en huracanes, determinación de potenciales de energía del oleaje o para caracterizar el clima marino de una región. Esta basado en la ecuación de balance de energía con fuentes y sumideros (parámetros que controlan el crecimiento o decrecimento del oleaje). El modelo por defecto usa mallas cartesianas o rectangulares para definir el dominio computacional, pero para este estudio se utiliza la malla no estructurada generada por el programa Gmsh mostrada en la Fig. 2. Al usar una malla triangular se tiene la libertad de definir aquellas regiones que requieran mayor resolución, como lo es, los alrededores de la isla y no todo el dominio computacional. Las coordenadas que abarca este estudio son $88^{\circ}-86^{\circ} \mathrm{W}$ y $4.5^{\circ}-6.5^{\circ} \mathrm{N}$. La batimetría para propagar el oleaje es proporcionada por el SIGMAR y Lizano (2012). El rango de frecuencias para el oleaje es de $0.0373-0.7159 \mathrm{~Hz}$ (incremento en frecuencia de forma logarítmica, $1.1 \mathrm{~Hz}$ ) y 24 direcciones en el espectro de energía del oleaje $\left(\Delta \theta=15^{\circ}\right)$. Se escoge en la configuración el esquema $\mathrm{N}$ para la propagación numérica (esquema explícito que se ajusta dinámicamente) (Csik, Ricchiuto \& Deconinck, 2002; Roland, 2008). Para el crecimiento y disipación 
del oleaje se utiliza la parametrización física de Ardhuin et al. (2010). Para las interacciones no lineales entre olas (cuadrupletas) se emplea el método aproximado de Hasselmann, Hasselmann, Allender \& Barnett (1985). Las condiciones iniciales son de calma (altura significante del oleaje de $0 \mathrm{~m}$ ) y las condiciones de frontera son dadas por los datos históricos de oleaje de IFREMER. No se emplea forzamiento debido al campo de viento pues solo se considera eventos de marejadas de fondo u oleaje largo ("swell", término en inglés). Del modelo de oleaje se extraen resultados de altura significante del oleaje (se define como el promedio de la tercera parte del oleaje con mayor altura), periodo (se define como el tiempo que transcurre al pasar una cresta o valle por un punto) y dirección dominante del oleaje (se define como la dirección de máxima energía del oleaje) son presentados para los alrededores de la Isla del Coco de acuerdo con el dominio mostrado en la Fig. 2 lado izquierdo. Para facilidad de interpretación los resultados de la dirección del oleaje se muestran usando una paleta de colores y no en su forma estandarizada de vectores (flechas). Estas variables son estadísticamente representativas de un intervalo de tiempo y son usadas para caracterizar el oleaje.

Estadísticos: Para evaluar el desempeño del modelo MIOCIMAR/WAVEWATCH III se aplica la siguiente métrica estadística a los resultados de altura significante del oleaje (Hs) y periodo asociado al pico espectral del oleaje (Tp): la diferencia en las medias (BIAS), la raíz media cuadrática (RMSE), el coeficiente de correlación (R) y el índice de dispersión (SI). Para los resultados de la dirección del oleaje dominante (Dp) se emplea la estadística para datos angulares de acuerdo con Mardia y Jupp (2009). Se calcula el promedio angular y la desviación estándar circular. Esta técnica es muy útil porque interpreta la dirección angular como vectores unitarios.

\section{RESULTADOS}

Comportamiento anual del campo de oleaje: El comportamiento anual de los resultados del modelo MIOCIMAR/WAVEWATCH III de la altura significante del oleaje, el periodo y la dirección correspondiente al pico del espectro de energía del oleaje se muestran en la Fig. 3. Cabe mencionar que la convención para la dirección del oleaje en los resultados sigue la convención meteorológica (de dónde viene). Se puede observar de la Fig. 3A que la altura significante del oleaje tiene un promedio entre 1.2 $\mathrm{m}$ y $1.4 \mathrm{~m}$ en las afueras de la isla. En la parte norte de la isla los promedios de altura del oleaje rondan entre los $0.6 \mathrm{~m}$ y $1.2 \mathrm{~m}$; notar que son menores que el promedio en las proximidades de la isla. En Bahía Wafer (de aquí en adelante referido como BW) (véase Fig. 2B) y Bahía Chatham (de aquí en adelante referido como $\mathrm{BC}$ ) (véase Fig. 2B) los promedios de altura significante del oleaje son entre $0.2 \mathrm{~m}$ y $0.6 \mathrm{~m}$.

Los periodos en las inmediaciones de la isla (Fig. 3B) son entre $13 \mathrm{~s} \mathrm{y} 14 \mathrm{~s}$. Estos periodos corresponden a un oleaje largo (oleaje de origen remoto con periodos mayores a 10 s) y son similares a los reportados en estudios previos (Young, 1999; Chen et al., 2002; Alves, 2006; Amador et al., 2006; Lizano, 2007; 2008). En la parte sur predomina una región con periodos entre los $14-15 \mathrm{~s}$ en el promedio anual. En BC los periodos son de $11 \mathrm{~s}$ en promedio igual que en BW.

En la parte oeste de la isla (afueras) la dirección predominante es del suroeste (Fig. 3C), mientras que en la parte este de la isla (afueras) la dirección del oleaje en promedio es del sur. En la parte noroeste, muy cerca de BW y Bahía Weston (de aquí en adelante referido como BWE; se localiza entre BW y BC), el oleaje es predominantemente de componente oeste - noroeste en el promedio anual. En BC predomina el oleaje con dirección noreste. En general, pareciera que $\mathrm{BC}$ es la zona más 


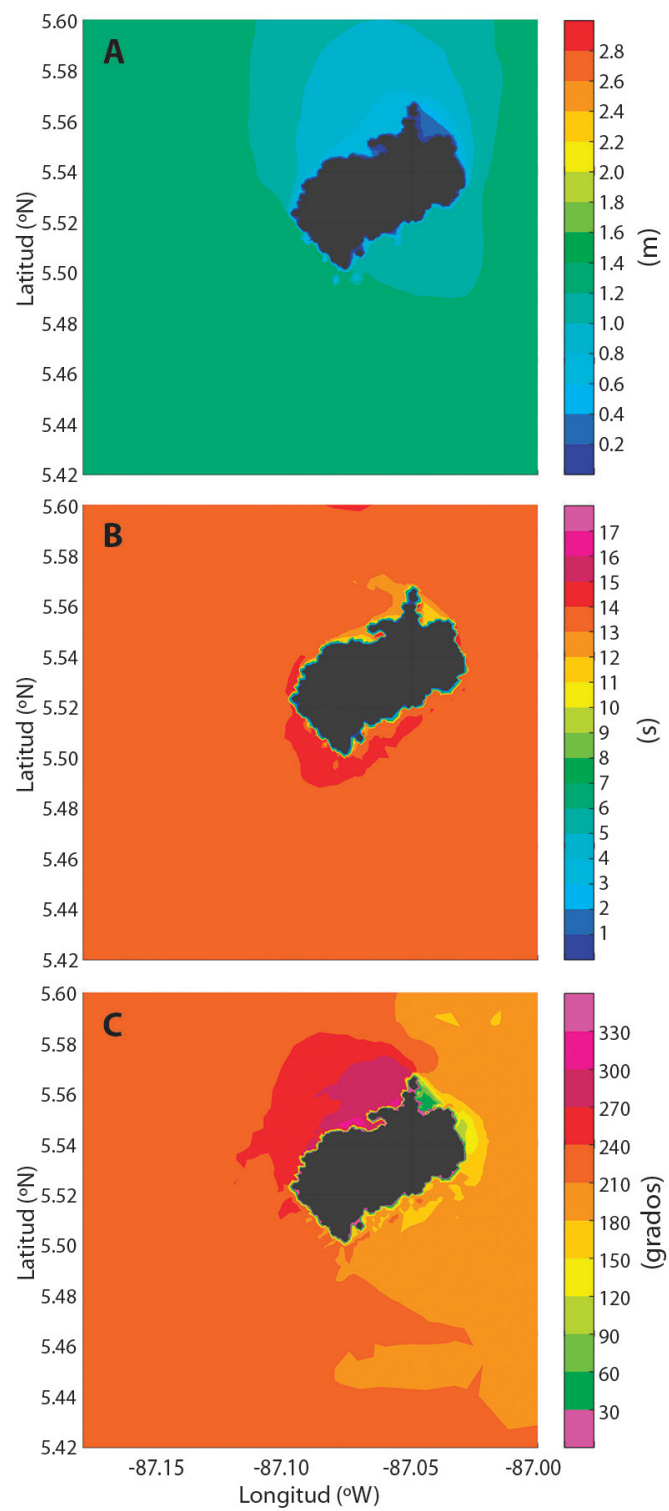

Fig. 3. Promedio anual del campo de oleaje en los alrededores de la Isla del Coco. En A) altura significante de la ola (m), B) periodo de la ola asociado al pico espectral (s) y C) dirección de la ola asociada al máximo del espectro (grados).

Fig. 3. Annual mean of the wave field in the surroundings of Cocos Island. In A) Significant wave height (m), B) peak wave period (s) and C) peak wave direction (degrees). protegida, presenta una menor altura de ola con respecto a $\mathrm{BW}$ y las proximidades de la isla.

\section{Comportamiento estacional del campo} de oleaje: Los resultados del comportamiento estacional de la altura del oleaje, el período y dirección del oleaje dominante del modelo MIOCIMAR/WAVEWATCH III se presentan en las Fig. 4, Fig. 5 y Fig. 6, respectivamente. En Fig. 4A, Fig. 5A, y Fig. 6A muestra el promedio estacional de los meses de diciembre a febrero (invierno boreal). Se observa que la altura significante del oleaje (Fig. 4A) en las inmediaciones de la isla el oleaje tiene una altura en promedio entre $1.0 \mathrm{~m}$ y $1.2 \mathrm{~m}$, mientras que en la parte norte y sur el oleaje es de altura menor, de $0.8 \mathrm{~m}$. En BW y BC las alturas del oleaje son menores a $0.6 \mathrm{~m}$. El periodo del pico espectral del oleaje en los alrededores de la isla es entre 13 s y 14 s (Fig. 5A), pero en la parte sur de la isla predomina una región con periodos entre $14 \mathrm{~s}$ y $15 \mathrm{~s}$ en el promedio estacional. En BC son notorios los periodos entre $8 \mathrm{~s}$ y $11 \mathrm{~s}$. La dirección predominante del oleaje (Fig. 6A) en la parte oeste de la isla es del suroeste-oeste, pero resalta la componente del oeste - noroeste en las cercanías de la isla (en la parte noroeste de la isla). En la región sureste de la isla el oleaje es de dirección proveniente del sur-suroeste en promedio.

Para los meses comprendidos entre marzo a mayo la altura significante del oleaje (Fig. 4B) presenta valores en promedio de $1.2 \mathrm{~m}$ y $1.4 \mathrm{~m}$ en los alrededores de la isla. Estos valores de altura del oleaje son ligeramente mayores al periodo dic-ene-feb. La altura del oleaje en la parte norte de la isla ronda entre $0.4 \mathrm{~m}$ y $1.0 \mathrm{~m}$. Se mantienen los valores de altura significante del oleaje en BW y BC igual que en el trimestre de dic-ene-feb. En cuanto a los periodos (Fig. 5B), en la mayor parte de las aguas adyacentes a la isla el promedio ronda entre 14 s y 15 s. Resalta una región al norte 

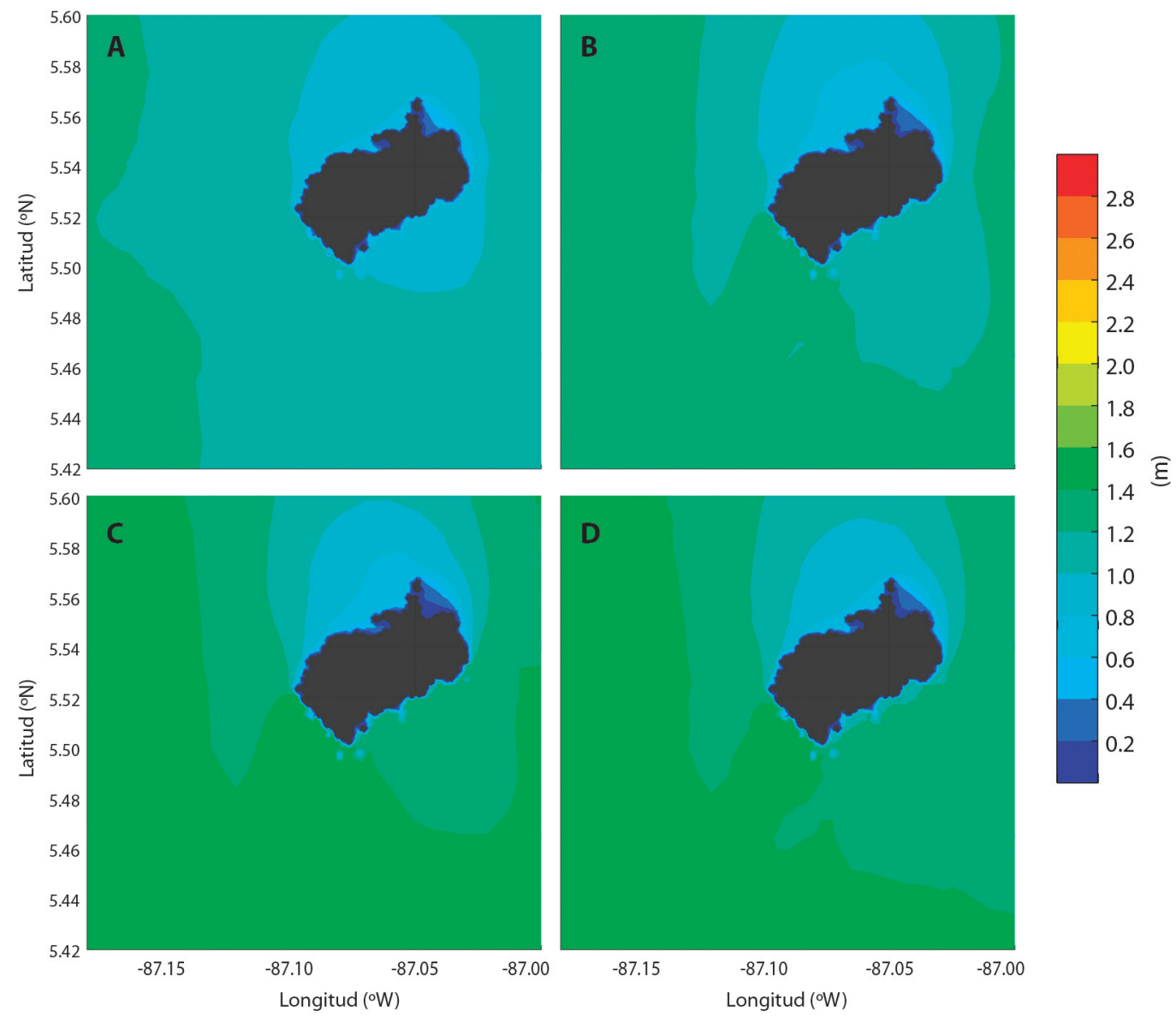

Fig. 4. Promedio estacional de la altura significante de la ola $(\mathrm{m})$ en los alrededores de la Isla del Coco. En A) diciembreenero-febrero, B) marzo-abril-mayo, c) junio-julio-agosto and D) septiembre-octubre-noviembre.

Fig. 4. Seasonal mean of significant wave height $(\mathrm{m})$ in the surroundings of Cocos Island. In A) December-JanuaryFebruary, B) March-May, C) June-July-August and D) September-October-November.

y noroeste de la isla con periodos entre $13 \mathrm{~s} \mathrm{y}$ $14 \mathrm{~s}$. Sobre BC los periodos en promedio son 12 - 13 s. La dirección predominante del oleaje muestra tres zonas de oleajes distintos (Fig. $6 \mathrm{~B}$ ), en la parte oeste predomina la componente del suroeste, mientras que en la parte este de la isla predomina la dirección proveniente del sur. En la región noroeste de la isla resalta el oleaje con componente oeste-noroeste. En BC el oleaje tiene una dirección en promedio proveniente del noreste.

Para el verano boreal (junio-julio-agosto) la altura significante del oleaje (Fig. 4C) en los alrededores de la isla aumenta su altura respecto a los trimestres anteriores (Fig. 4A, Fig. 4B), en promedio ronda los $1.4-1.6 \mathrm{~m}$. En la parte norte de la isla se mantiene el promedio de alturas de oleaje $(0.4-1.0 \mathrm{~m})$ con respecto al trimestre mar-abr-may (Fig. 4B), pero disminuye el área donde se presentan tales alturas de oleaje. En la mayor parte de la isla los períodos son entre $13 \mathrm{~s}$ y $14 \mathrm{~s}$ (Fig. 5C). Notar al oeste de la isla una región con periodos entre $11 \mathrm{~s}$ y $12 \mathrm{~s}$, misma que se presenta al frente de BWE. En las afueras de $\mathrm{BC}$ una región con periodos de 14 $15 \mathrm{~s}$. Sobre la isla es distinguible varias zonas con oleajes de distintas direcciones (Fig. 6C). Una gran región con dirección sur - suroeste 

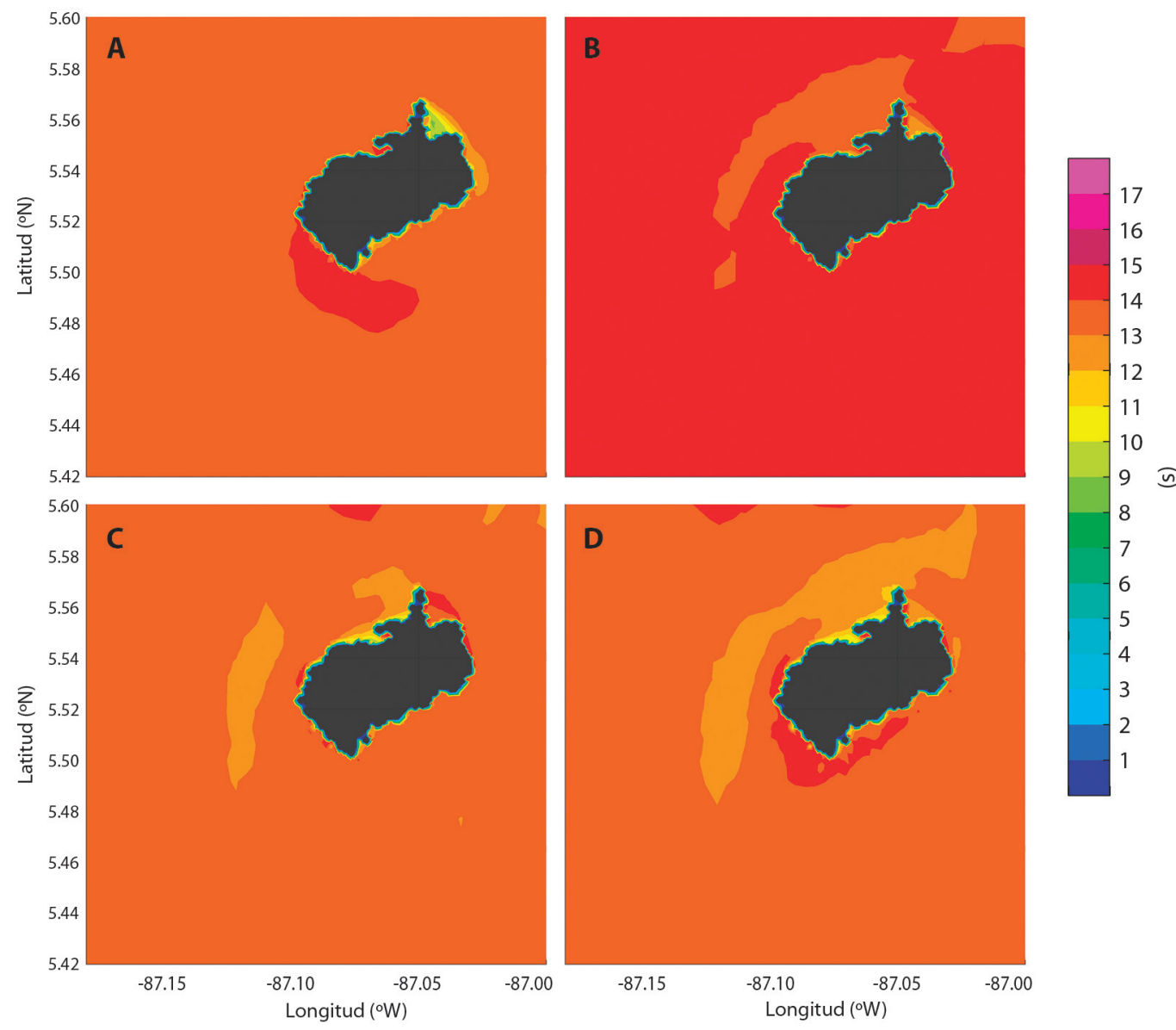

Fig. 5. Promedio estacional para el periodo asociado al pico del espectro de la ola (s) en los alrededores de la Isla del Coco. En A) diciembre-enero-febrero, B) marzo-abril-mayo, c) junio-julio-agosto and D) septiembre-octubre-noviembre..

Fig. 5. Seasonal mean for the peak wave period (s) in the surroundings of Cocos Island. In A) December-January-February, B) March-May, C) June-July-August and D) September-October-November.

en promedio que va desde el noroeste hasta el sureste hacia las afueras de la isla. Hacia la parte noreste la dirección predominante es del sur- sureste, mientras en la parte noroeste, muy cerca de la isla, la dirección que prevalece proviene del oeste en promedio. Sobre BC la componente del oleaje proveniente del noreste es la que domina.

La estación comprendida entre septiembre a noviembre muestra que la altura del oleaje (Fig. 4D) sobre la parte norte de la isla mantiene valores similares $(0.4-1.0 \mathrm{~m})$ al trimestre anterior (jun-jul-ago), pero el área se reduce con respecto a la Fig. 4C. En los alrededores de la isla la altura significante del oleaje promedio ronda entre $1.2 \mathrm{~m}$ y $1.6 \mathrm{~m}$. Sobre BC y BW se mantienen los valores promedio menores a $0.8 \mathrm{~m}$. Los periodos (Fig. 5D) entre 13 y 14 $\mathrm{s}$ predominan en los alrededores y lejos de la isla. Es notorio una zona con periodos de 12 -13 s que bordea la isla desde la parte suroeste hasta la parte noreste de esta. En la parte sur predomina una región con periodo mayora 14 s. La dirección dominante de las olas promedio se muestra en la Fig. 6D. Se evidencian tres zonas con dirección del oleaje diferente, una en 

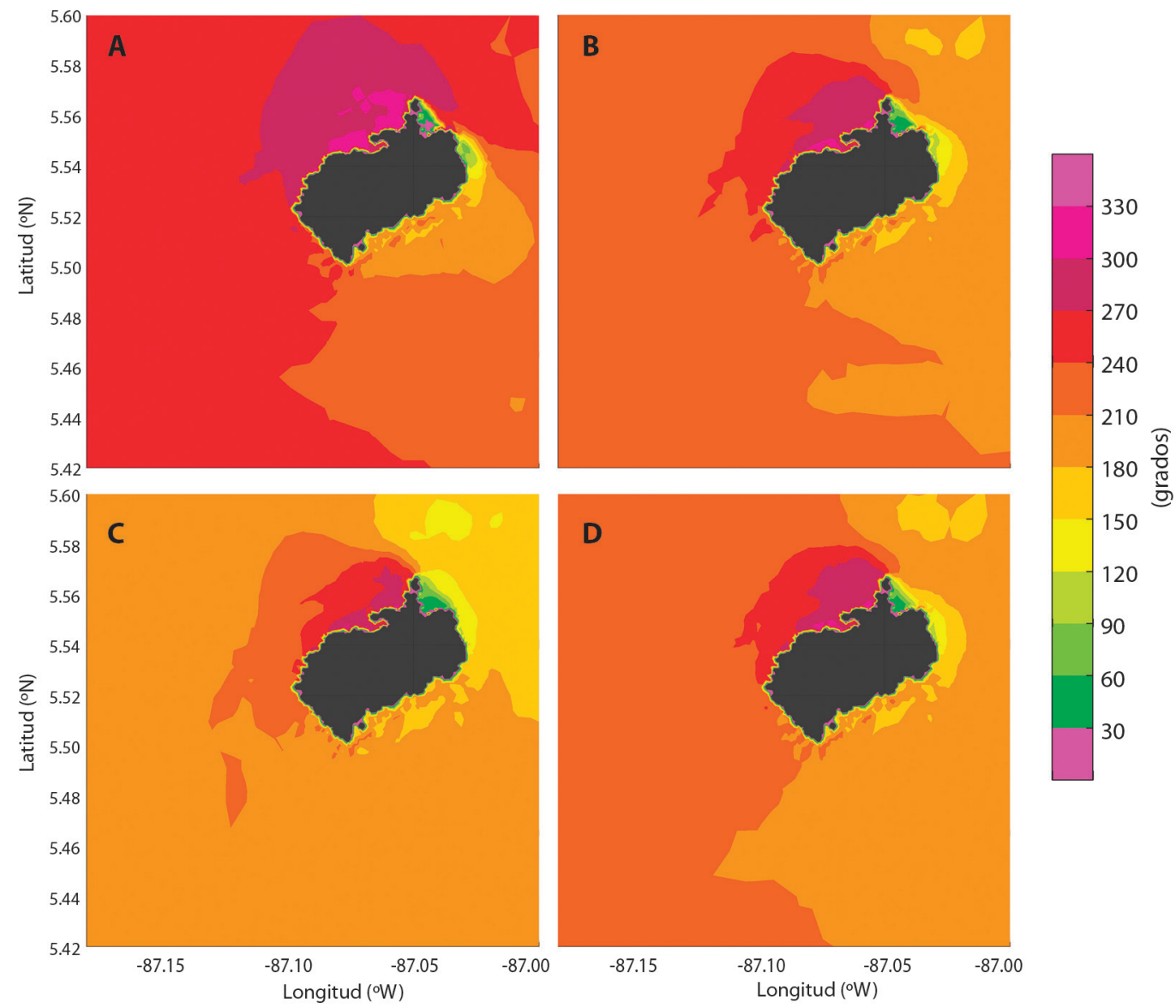

Fig. 6. Promedio estacional para la dirección dominante de la ola (grados) en los alrededores de la Isla del Coco. En A) diciembre-enero-febrero, B) marzo-abril-mayo, c) junio-julio-agosto and D) septiembre-octubre-noviembre.

Fig. 6. Seasonal mean for the peak wave direction (degrees) in the surroundings of Cocos Island. In A) December-JanuaryFebruary, B) March-May, C) June-July-August and D) September-October-November.

la parte oeste y este de las inmediaciones de la isla con dirección proveniente del sur, mientras en la parte noroeste predomina oleaje del oeste. En este periodo como en los anteriores (Fig. 6A, Fig. 6B, Fig. 6C) prevalece oleaje desde el noreste en $\mathrm{BC}$.

Oleaje en un punto cercano a la Isla del Coco: En la Fig. 7 se muestra la serie temporal de los resultados del modelo MIOCIMAR/WAVEWATCH III para el punto $-87.5^{\circ}$ $\mathrm{W}-5.5^{\circ} \mathrm{N}$. Para referencia en la figura se muestra los datos históricos del modelo operacional NOAA/WAVEWATCH III para la misma coordenada. Este es un modelo ampliamente validado alrededor del mundo. Una de las razones para hacer esta comparación es la ausencia de equipo oceanográfico in situ como boyas oceanográficas cercanas a la isla o la utilización de la información de altímetros ya que son dispersos y distantes a la zona de estudio. Entre los meses de enero y marzo se observa la predominancia de la dirección proveniente del oeste (Fig. 7C) y el oleaje es de menor altura (Fig.7A), similar a los datos históricos de NOAA/WAVEWATCH III. Ambos modelos muestran que la dirección dominante del oleaje (Fig. 7C) en los meses de diciembre y enero 

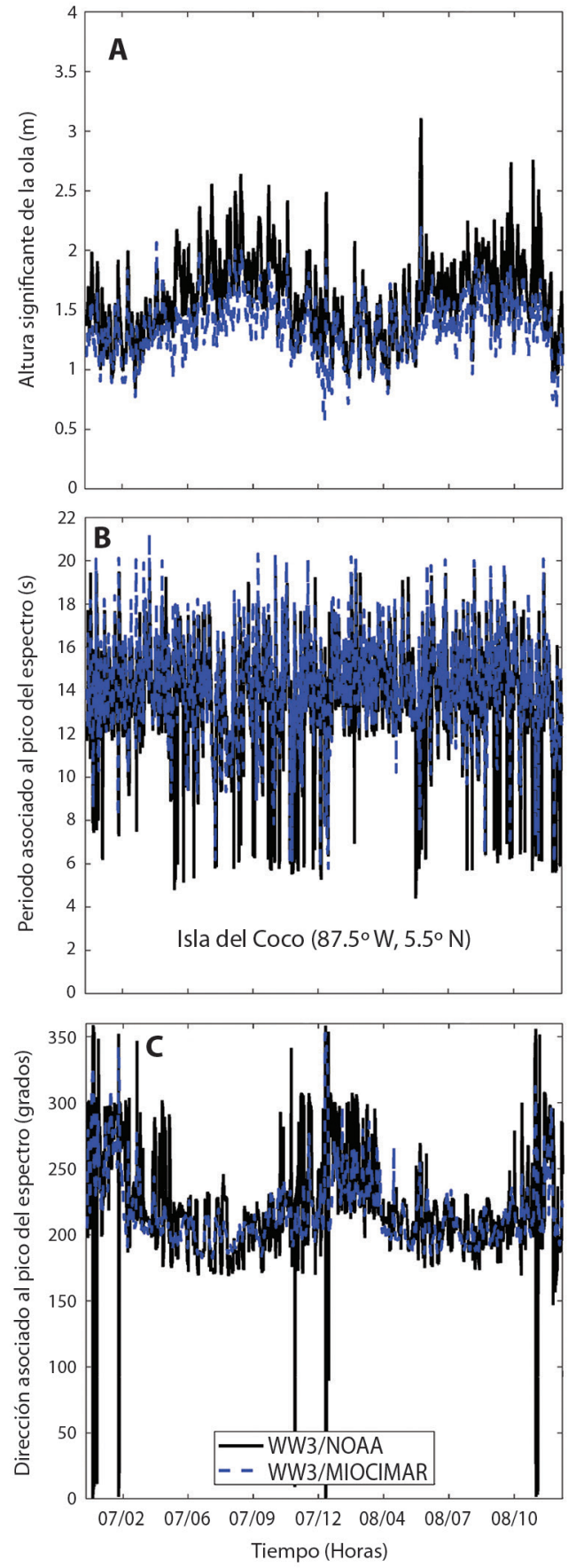

proviene del noroeste, esto es consistente con los resultados de Young (1999), Amador et al. (2006) y Lizano (2007; 2008).

Entre abril y noviembre, predominan las marejadas de fondo provenientes del suroeste (Fig.7C) en ambos modelos, siendo esta la dirección promedio del oleaje que arriba a la Isla del Coco y consistente con lo reportado en la literatura (Young, 1999; Chen et al., 2002; Alves, 2006; Amador et al., 2006; Lizano, 2007, 2008). Para este periodo se nota que la altura significante del oleaje aumenta en altura comparado con los demás meses, este resultado es similar a lo reportado por Lizano (2008).

En el Tabla 1 se presenta la estadística para la altura significante del oleaje y el periodo asociado al pico del espectro del oleaje. La altura significante del oleaje presenta un RMSE bajo $(0.29 \mathrm{~m})$ y una correlación positiva $(0.84)$. El índice de dispersión es bajo $(0.11 \mathrm{~m})$ lo que indica que el porcentaje de error del modelo MIOCIMAR/WAVEWATCH III es menor respecto al modelo de la NOAA/WAVEWATCH III. El BIAS es negativo $(-0.23 \mathrm{~m})$, lo que indica que los valores de la altura del oleaje son ligeramente subestimados en el modelo MIOCIMAR/WAVEWATCH III. En el periodo del oleaje la métrica estadística da una correlación de 0.54 , el RMSE es de 2.47 s y un BIAS muy bajo, de $0.70 \mathrm{~s}$. El índice de dispersión es bajo, de $0.18 \mathrm{~s}$.

La estadística para la dirección dominante del oleaje se muestra en el Tabla 2. El promedio de la dirección del oleaje indica que ambos modelos la dirección es del suroeste $\left(215.63^{\circ}\right.$ y $\left.221.26^{\circ}\right)$. La dirección dominante del oleaje presenta una dispersión angular baja, siendo menor en el modelo MIOCIMAR/ WAVEWATCH III $\left(0.44^{\circ}\right)$ que en el modelo de la NOAA/WAVEWATCH III $\left(0.63^{\circ}\right)$.

Fig. 7. Serie temporal de: A) altura significante del oleaje, B) periodo de la ola asociado al pico del espectro y C) dirección asociada al pico espectral para los años 2007 y 2008 . El punto es $87.5^{\circ} \mathrm{W}-5.5^{\circ} \mathrm{N}$. La línea sólida negra representa el modelo de la NOAA y la línea discontinua azul representa el modelo MIOCIMAR.

Fig. 7. Time series of: A) significant wave height, B) peak wave period and C) wave peak for the years 2007 and 2008. The point is $87.5^{\circ} \mathrm{W}-5.5^{\circ} \mathrm{N}$. The black solid black line represents the NOAA model and the blue dashed line represents MIOCIMAR model. 
TABLA 1

Estadística descriptiva para la altura significante del oleaje (Hs) y el periodo del oleaje asociado al pico del espectro de energía (Tp).

TABLE 1

Descriptive statistics for significant wave height (Hs) and peak wave period (Tp).

\begin{tabular}{ccccc} 
Variable & BIAS & RMSE & R & SI \\
$\mathrm{Hs}$ & $-0.23 \mathrm{~m}$ & $0.29 \mathrm{~m}$ & 0.84 & $0.11 \mathrm{~m}$ \\
$\mathrm{Tp}$ & $0.70 \mathrm{~s}$ & $2.47 \mathrm{~s}$ & 0.54 & $0.18 \mathrm{~s}$ \\
\hline
\end{tabular}

TABLA 2

Estadística circular para la dirección de la ola correspondiente al pico del espectro de energía (Dp).

TABLE 2

Circular statistics for the peak wave direction (Dp).

\begin{tabular}{lcc}
\multicolumn{1}{c}{ Modelo } & Dp promedio & Dp desviación estándar \\
MIOCIMAR/WAVEWATCH & $215.63^{\circ}$ & $0.44^{\circ}$ \\
NOAA/WAVEWATCH & $221.26^{\circ}$ & $0.63^{\circ}$ \\
\hline
\end{tabular}

Oleaje en las cercanías de Bahía Wafer: Un punto de interés en la Isla del Coco es la Bahía Wafer (noroeste de la isla, véase Fig. 1) que se utiliza para la llegada y salida de embarcaciones. Otro sitio de arribo a la isla es la Bahía Chatham (noreste de la isla, véase Fig. 1). Es importante mencionar que ambos sitios son utilizados para el atraque de embarcaciones pequeñas o medianas, pero este arribo depende de las características del oleaje presentes, según testimonios de los guardaparques de la isla (comunicación personal, I Simposio Internacional sobre la Isla del Coco, 2018). Para mostrar lo que afirman los guardaparques de la Isla del Coco, en la Fig. 8 se presenta la serie temporal de la altura del oleaje, periodo y dirección correspondiente al pico espectral del oleaje para un punto cercano a Bahía Wafer. La altura significante del oleaje en esta zona tiende a disminuir cuando la dirección asociada al pico espectral del oleaje pierde componente del norte, con más predominancia de oleaje desde el oeste (oeste-noroeste) (Fig. 8A, Fig. 8B). $\mathrm{La}$ altura promedio del oleaje en este punto, es cercano a los $0.7 \mathrm{~m}$. Esta región mantiene un periodo del oleaje en promedio de alrededor de los 15 s como se indica en la línea roja punteada de la Fig. 8C. Los meses del invierno boreal (noviembre - febrero) predomina el oleaje con componente norte (Fig. 8C), mientras los meses del verano boreal (junio - septiembre) la dirección dominante del oleaje es del suroeste (Fig. 8C). La dirección promedio del oleaje es alrededor de los $303^{\circ}$.

\section{DISCUSIÓN}

El comportamiento anual del campo de oleaje nos indica que en la mayor parte de la isla el oleaje proviene del sur-suroeste, con periodos largos ( $>13 \mathrm{~s}$ ) y alturas de olas de $1.2 \mathrm{~m}$. Este oleaje característico es generado remotamente en las tormentas extratropicales del Hemisferio Sur (Young, 1999; Amador et al., 2006; Lizano, 2007; 2008; 2017). Young (1999) menciona que las marejadas de fondo que alcanzan el PTE son generadas en la zona intensa de oleaje del Océano del Sur entre Australia y Sudáfrica (Lizano, 2017). Al ser una región sin obstáculos (continentes) el oleaje escapa de la zona de generación y viaja libremente a través del océano Pacífico (Alves, 2006), inclusive alcanzando la costa oeste de Estados Unidos. Este oleaje de origen remoto 

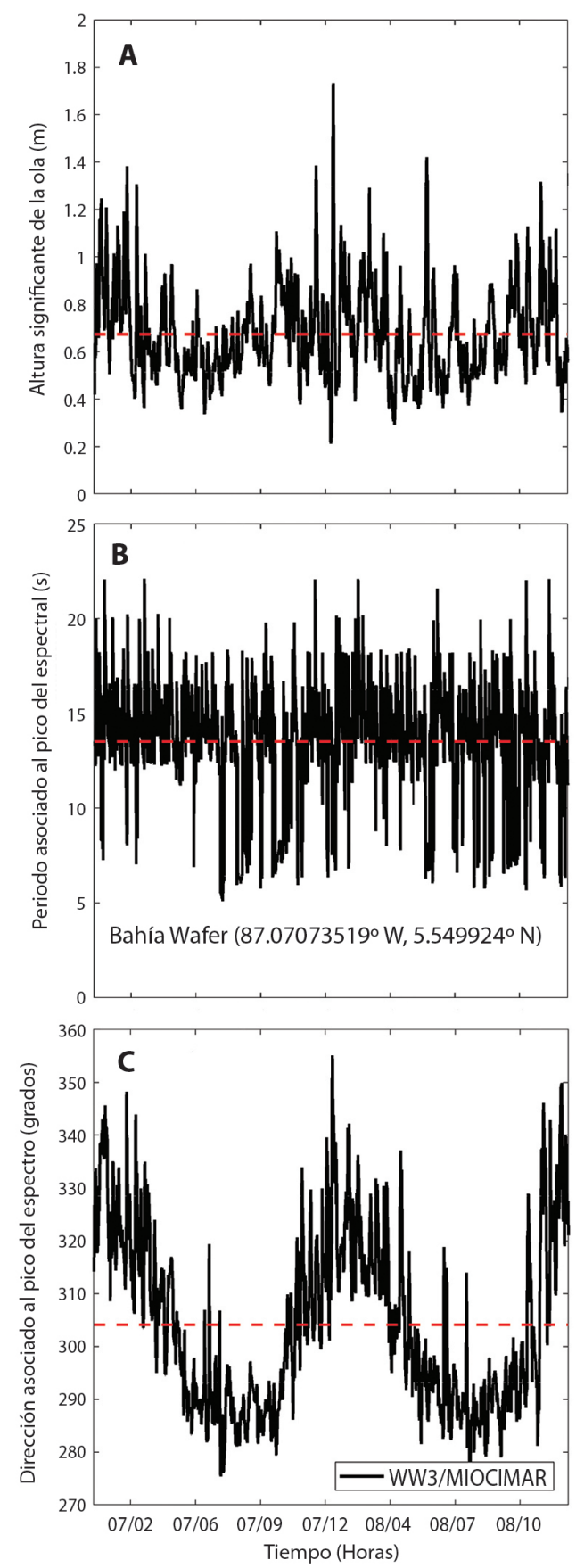

está presente casi todo el año (Young, 1999; Alves, 2006). En la climatología de oleaje de Lizano (2007) con datos de reanálisis de NCEP, su análisis muestra que el oleaje que arriba a las costas del Pacífico de Costa Rica en la mayor parte del año es del suroeste, es decir, marejadas de fondo. Cabe resaltar que este oleaje pasa primero por la Isla del Coco.

Cuando se analiza el campo de oleaje por estaciones del año en las cercanías de la isla, es evidente de los resultados (Figs. 4, 5, 6) que las características del oleaje son moduladas por los inviernos del Hemisferio Norte y Hemisferio Sur (Amador et al., 2006; Lizano 2007, 2008, 2017). En el invierno boreal los vientos alisios soplan sobre el Mar Caribe, se extienden e intensifican hasta el Pacífico a través de los pasos montañosos al norte de Costa Rica (Amador et al., 2006; Amador et al., 2016; Lizano, 2017). Este patrón de viento modifica la marejada de fondo presente en los alrededores de la isla disminuyendo la altura de oleaje como se mostró en la Fig. 4A, semejante a los resultados obtenidos por Lizano (2007, 2008). A pesar de que en promedio la dirección dominante del oleaje es del suroeste, en la parte noroeste de la isla la componente noroeste del oleaje resalta, este patrón es consistente con los resultados de Amador et al. (2006) y Lizano (2008, 2017). Precisamente son marejadas de fondo generadas de tormentas intensas en el Pacífico Norte y que su influencia en la región persiste alrededor de 6 meses (Alves, 2006).

Para el verano boreal (invierno en el Hemisferio Sur) los resultados de la simulación de la altura significante del oleaje ha incrementado en promedio a $1.5 \mathrm{~m}$, con periodos de 13 $\mathrm{s}$ y la dirección dominante es del sur-suroeste, esto confirma lo mencionado en estudios similares (Young, 1999; Amador et al., 2006; Lizano, 2007, 2008, 2017). Este oleaje es generado en las tormentas extratropicales del Hemisferio

Fig. 8. Serie temporal de: A) altura significante del oleaje, B) periodo de la ola asociado al pico del espectro y C) dirección asociada al pico espectral para un punto representativo en Bahía Wafer $\left(87.07073519^{\circ} \mathrm{W}-5.549924^{\circ} \mathrm{N}\right)$. Comprende los años 2007 y 2008. La línea sólida negra representa el modelo de WW3/MIOCIMAR. La línea discontinua roja indica el promedio (A-B) y promedio angular (C).

Fig. 8. Time series of: A) significant wave height B) peak wave period and C) peak wave direction for a representative point at Wafer Bay $\left(87.07073519^{\circ} \mathrm{W}-5.549924^{\circ} \mathrm{N}\right)$. The time span is two years $(2007-2008)$. The black solid line represents the WW3/MIOCIMAR model. The red dashed line indicates the mean (A-B) and angular mean (C). 
Sur (Young, 1999; Alves, 2006; Amador et al., 2006; Lizano, 2007, 2008, 2017). Es interesante notar que los periodos mayores de oleaje se dan en la primavera (Fig. 5B). Young (1999) explica que estos periodos largos se deben a un debilitamiento de los sistemas de baja presión en el Pacífico Norte y que los sistemas extratropicales del Hemisferio Sur se refuerzan. En $\mathrm{BC}$ se mantiene durante todo el año la dirección proveniente del noreste, esto se debe al proceso de refracción del oleaje que viene en promedio del sur - suroeste.

La comparación con el modelo operacional de pronóstico NOAA/WAVEWATCH III indica que los resultados para el punto $87.5^{\circ} \mathrm{W}$ $-5.5^{\circ} \mathrm{N}$ tiene una alta correlación con la altura significante del oleaje y valores bajos de RMSE y SI. El periodo tiene una correlación de $0.54 \mathrm{~s}$. Este valor bajo de $\mathrm{R}$ en el periodo comparado con el valor alto obtenido para la altura significante de la ola, se relaciona con el hecho de que el modelo MIOCIMAR/WAVEWATCH III no es forzado por un campo de viento. Se observa en la Fig. 7B los periodos cortos que presenta el modelo de la NOAA (5 - $6 \mathrm{~s}$ ), esto indica la generación de oleaje local. La dirección dominante en promedio es del suroeste, resultado esperado y que confirma lo reportado en la literatura (Young, 1999; Chen et al., 2002, Alves, 2006, Amador et al., 2006, Lizano, 2007; 2008; 2017). La desviación angular es baja en ambos modelos. En general el modelo MIOCIMAR/ WAVEWATCH III tiene un buen desempeño de acuerdo con las métricas utilizadas.

Bahía Wafer es un sitio utilizado para el arribo de las embarcaciones al Parque Nacional Isla del Coco. Dado que es un lugar frecuente para el atraque a la isla, en la Fig. 8 se mostró la serie temporal de Hs, Tp y Dp. Los resultados son congruentes con lo mostrado en la Fig. 7. Es notorio de la figura que el oleaje en la región se comporta de acuerdo con el patrón atmosférico de los sistemas extratropicales (Amador et al., 2006; Lizano 2007, 2008, 2017), es decir, en el verano boreal se presentan menores alturas de oleaje y la dirección dominante del oleaje es del oeste, mientras que en el invierno boreal la dirección del oleaje da un giro de aproximadamente $60^{\circ}$ (dirección noroeste en promedio). La altura significante del oleaje es ligeramente mayor que en el periodo de diciembre a febrero. Persisten en promedio los periodos alrededor de 13 s. Estos resultados confirman los testimonios de los guardaparques (comunicación personal, 2018), de que el desembarco en Bahía Wafer o en Bahía Chatham depende de las condiciones de oleaje y la época del año en que se visita la Isla del Coco.

Otro punto importante para recalcar son las implicaciones que tiene el conocer las características del oleaje en procesos biológicos, por ejemplo, la Isla del Coco es conocida por su biodiversidad marina, específicamente su fauna coralina. La isla posee la mayor longitud de arrecifes de coral de Costa Rica, alrededor de $2 \mathrm{~km}$ (Glynn et al., 2017). Esta comunidad de corales que se ubica en la parte norte de la isla presenta mejores condiciones de crecimiento según Glynn et al. (2017). Una de las razones que se argumentan para el desarrollo del arrecife coralino es que por su ubicación se protege del oleaje que proviene del sur, de acuerdo con lo reportado en literatura (Guzmán \& Cortés, 1992; Cortés, 2016). Este estudio numérico evidencia que la dirección predominante del oleaje en la Isla del Coco en promedio es sursuroeste, por lo que inferimos que el sector norte de la isla sea una zona ideal para una gran diversidad de ecosistemas.

La simulación de oleaje sobre los alrededores de la Isla del Coco implementando el modelo MIOCIMAR/WAVEWATCH III con una malla no estructurada mostró que representa las condiciones típicas del oleaje prevalecientes en el PTE. Estos resultados pueden servir de base para posteriores estudios en la Isla del Coco y planificar las actividades humanas que se desarrollan cerca de la isla. El modelo MIOCIMAR/ WAVEWATCH III puede replicarse a otras áreas específicas de la costa de Costa Rica.

Declaración de ética: los autores declaran que todos están de acuerdo con esta publicación y que han hecho aportes que justifican su autoría; que no hay conflicto de interés de ningún tipo; y que han cumplido con todos los 
requisitos y procedimientos éticos y legales pertinentes. Todas las fuentes de financiamiento se detallan plena y claramente en la sección de agradecimientos. El respectivo documento legal firmado se encuentra en los archivos de la revista.

\section{AGRADECIMIENTOS}

Se agradece al siguiente proyecto inscrito en la Vicerrectoría de Investigación, Universidad de Costa Rica: 808-A7-166. A la Vicerrectoría de Investigación y CIMAR por el tiempo de apoyo. Al Coloboratorio Nacional Computación Avanzada (CNCA) del Centro Nacional de Alta Tecnología (CENAT) por los servicios en la plataforma computacional. Al SIGMARCIMAR por brindar los datos de batimetría y línea de costa en la Isla del Coco. En la edición de imágenes el apoyo de Mauricio Lao Escalante. A Omar Lizano y revisores anónimos por su comentarios y sugerencias para mejorar el presente trabajo.

\section{RESUMEN}

Introducción: La Isla del Coco por su ubicación en el océano Pacífico y en aguas profundas, periódicamente arriban olas energéticas que se originan en el Océano del Sur. La isla actúa como un abrigo disipando parte de la energía de las olas que llegan a la costa pacífica de Costa Rica. Objetivo: El objetivo del trabajo es caracterizar las condiciones generales del oleaje a través de la simulación numérica del oleaje en el Pacífico Tropical del Este (PTE), con especial interés en la Isla del Coco. Métodos: Las olas se propagan con el modelo espectral de oleaje WAVEWATCH III. Se usa en el modelo una malla no estructurada. Se utilizan dos años (2007-2008) de datos históricos de oleaje como condiciones de frontera obtenidos de reanálisis con el modelo WAVEWATCH generado por el Instituto Francés para la Investigación del Mar (IFREMER por sus siglas en francés). Se obtienen mapas anuales y estacionales y series temporales de la altura significante de la ola, periodo y dirección asociado al pico del espectro de energía. Resultados: Los resultados mostrados son similares a otros estudios previos de simulación y observación. El campo de oleaje es caracterizado por periodos largos y dirección desde suroeste en promedio. Las condiciones de oleaje sobre la isla obedecen a los sistemas extratropicales de ambos hemisferios y a procesos locales en el PTE. Conclusiones: El modelo de olas WAVEWATCH III mostró que representa las condiciones típicas de oleaje en los alrededores de la Isla del Coco. Es el primer trabajo de simulación de oleaje aplicando una malla no convencional en la zona económica exclusiva de Costa Rica. El estudio sirve como base para extenderse a otras áreas específicas de la costa.

Palabras clave: altura significante de la ola; generador de mallas no estructuradas; Isla del Coco; marejada de fondo; Pacífico Tropical del Este; WAVEWATCH III.

\section{REFERENCIAS}

Avdis, A., Candy, A. S., Hill, J., Kramer, S. C., \& Piggott, M. D. (2018). Efficient unstructured mesh generation for marine renewable energy applications. Renewable Energy, 116, 842-856.

Alves, J. H. G. (2006). Numerical modeling of ocean swell contributions to the global wind-wave climate. Ocean Modelling, 11(1-2), 98-122.

Amador, J. A., Alfaro, E. J., Lizano, O. G., \& Magaña, V. O. (2006). Atmospheric forcing of the eastern tropical Pacific: A review. Progress in Oceanography, 69(24), 101-142.

Amador, J. A., Durán-Quesada, A. M., Rivera, E. R., Mora, G., Sáenz, F., Calderón, B., \& Mora, N. (2016). The easternmost tropical Pacific. Part II: Seasonal and intraseasonal modes of atmospheric variability. Revista de Biología Tropical, 23-57.

Ardhuin, F., Rogers, E., Babanin, A. V., Filipot, J. F., Magne, R., Roland, A...Collard, F. (2010). Semiempirical dissipation source functions for ocean waves. Part I: Definition, calibration, and validation. Journal of Physical Oceanography, 40(9), 1917-1941.

Ardhuin, F., \& Roland, A. (2012). Coastal wave reflection, directional spread, and seismoacoustic noise sources. Journal of Geophysical Research: Oceans, 117(11).

Booij, N., Holthuijsen, L. H., \& Ris, R. C. (1996). The" SWAN" wave model for shallow water. Coastal Engineering Proceedings, 1(25).

Csık, A., Ricchiuto, M., \& Deconinck, H. (2002). A conservative formulation of the multidimensional upwind residual distribution schemes for general nonlinear conservation laws. Journal of computational physics, 179(1), 286-312.

Chen, G., Chapron, B., Ezraty, R., \& Vandemark, D. (2002). A global view of swell and wind sea climate in the ocean by satellite altimeter and scatterometer. Journal of Atmospheric and Oceanic Technology, 19(11), 1849-1859, DOI: doi.org/10.1175/15200426 (2002)019<1849:AGVOSA>2.0.CO;2

Cortés, J. (2016). The Pacific coastal and marine ecosystems. In M. Kapelle (Ed.), Costa Rican Ecosystems (pp. 97-138). Chicago and London: University of Chicago Press.

Dietrich, J. C., Tanaka, S., Westerink, J. J., Dawson, C. N., Luettich, R. A., Zijlema, M., ... \& Westerink, H. J. (2012). Performance of the unstructured-mesh, SWAN+ ADCIRC model in computing hurricane 
waves and surge. Journal of Scientific Computing, $52(2), 468-497$.

Geuzaine, C., \& Remacle, J. F. (2009). Gmsh: a threedimensional finite element mesh generator with builtin pre- and post-processing facilities. International Journal for Numerical Methods in Engineering, 79(11), 1309-1331.

Glynn, P. W., Alvarado, J. J., Banks, S., Cortés, J., Feingold, J. S., Jiménez, C., ... \& Navarrete, S. (2017). Eastern Pacific coral reef provinces, coral community structure and composition: an overview. In P. W. Glynn, P. Manzello, \& I. C. Enochs (Eds.), Coral Reefs of the Eastern Tropical Pacific, (pp. 107-176). Dordrecht, Netherlands: Springer.

Guzmán, H. M., \& Cortés, J. (1992). Cocos Island (Pacific of Costa Rica) coral reefs after the 1982-83 El Niño disturbance. Revista de Biología Tropical, 40(3), 309-324.

Hasselmann, S., Hasselmann, K., Allender, J. H., \& Barnett, T. P. (1985). Computations and parameterizations of the nonlinear energy transfer in a gravity-wave specturm. Part II: Parameterizations of the nonlinear energy transfer for application in wave models. Journal of Physical Oceanography, 15(11), 1378-1391.

Kessler, W. S. (2006). The circulation of the eastern tropical Pacific: A review. Progress in Oceanography, 69(2-4), 181-217.

Lizano, O. G. \& Moya, R. J. (1990). Simulación de oleaje durante el huracán Joan (1988) a su paso por el mar Caribe de Costa Rica. Revista Geofisica, (33), 105-126.

Lizano, O. G. (2001). Batimetría de la plataforma insular alrededor de la Isla del Coco, Costa Rica, Centro América. Revista de Biología Tropical, 49 (Suplemento 2), 163-170.

Lizano, O. G., Ocampo, F. J., Alvarado, L. F., Vega, R. \& Puig, J. M. (2001). Evaluación de modelos numéricos de Tercera Generación para el pronóstico del oleaje en Centroamérica y México. Tópicos Meteorológicos y Oceanográficos, 8(1), 40-49.

Lizano, O. G. (2007). Climatología del viento y oleaje frente a las costas de Costa Rica. Ciencia y Tecnología, 25(1-2), 43-56.

Lizano, O. G. (2008). Dinámica de las aguas alrededor de la Isla del Coco, Costa Rica. Revista de Biología Tropical, 56(Suplemento 2), 31-48.

Lizano, O. G. (2012). Rasgos morfológicos alrededor de la Isla del Coco y de sus montes submarinos vecinos, Pacífico de Costa Rica. Revista de Biología Tropical, 60(Suplemento 3), 43-51.

Lizano, O. G. (2017). Los accidentes acuáticos alrededor de nuestras costas y su relación con el estado del mar y de la atmósfera. Revista En Torno a la Prevención, 18, 07-14.
Luettich, R. A., \& Westerink, J. J. (2004). Formulation and numerical implementation of the $2 D / 3 D$ ADCIRC finite element model version 44. XX, 74. Retrieved from http://www.twdb.texas.gov/publications/ reports/contracted_reports/doc/2002483450.pdf

Mardia, K. V., \& Jupp, P. E. (2009). Directional statistics (Vol. 494). London, UK: John Wiley \& Sons, Ltd.

Munk, W. H., \& Snodgrass, F. E. (1957). Measurements of southern swell at Guadalupe Island. Deep Sea Research (1953), 4, 272-286.

Remacle, J. F., \& Lambrechts, J. (2016). Fast and robust mesh generation on the sphere-application to coastal domains. Procedia engineering, 163, 20-32.

Rascle, N., \& Ardhuin, F. (2013). A global wave parameter database for geophysical applications. Part 2: Model validation with improved source term parameterization. Ocean Modelling, 70, 174-188.

Roland, A. (2008). Development of WWM II Model: Spectral wave modelling on unstructured meshes. (Doctoral dissertation). Darmstadt University of Technology, Institute of Hydraulic and Water Resources Engineering, Germany.

Rojas, W., \& Alvarado, G. E. (2012). Geología y contexto geotectónico de la Isla del Coco y la zona marítima frente al Pacífico central de Costa Rica. Revista de Biología Tropical, 60(Suplemento 3), 15-32.

Snodgrass, F. E., Hasselmann, K. F., Miller, G. R., Munk, W. H., \& Powers, W. H. (1966). Propagation of ocean swell across the Pacific. Philosophical Transactions of the Royal Society of London. Series A, Mathematical and Physical Sciences, 259(1103), 431-497.

Shewchuk, J. R. (1996, May). Triangle: Engineering a 2D quality mesh generator and Delaunay triangulator. In M. C. Lin \& D. Manocha (Eds.), Applied computational geometry towards geometric engineering (pp. 203-222). Berlin, Germany: Springer.

Tolman, H. (2014). User Manual and system documentation of WAVEWATCH III version 4.18. Environmental Modeling Center, Marine Modeling and Analysis Branch.

Warren, I. R., \& Bach, H. (1992). MIKE 21: a modelling system for estuaries, coastal waters and seas. Environmental Software, 7(4), 229-240.

Wyrtki, K. (1967). Circulation and water masses in the eastern equatorial Pacific Ocean. International Journal of Oceanology and Limnology, 1, 117-147.

Young, I. R. (1999). Seasonal variability of the global ocean wind and wave climate. International Journal of Climatology, 19(9), 931-950.

Zijlema, M. (2010). Computation of wind-wave spectra in coastal waters with SWAN on unstructured grids. Coastal Engineering, 57(3), 267-277. 